\title{
Probing Massive Star Nucleosynthesis with Data on Metal- Poor Stars and the Solar System
}

\author{
Yong-Zhong Qian ${ }^{1, *}$ \\ ${ }^{1}$ School of Physics and Astronomy, University of Minnesota, Minneapolis, Minnesota 55455, USA
}

\begin{abstract}
Metal-poor stars were formed during the early epochs when only massive stars had time to evolve and contribute to the chemical enrichment. Low-mass metal-poor stars survive until the present and provide fossil records of the nucleosynthesis of early massive stars. On the other hand, short-lived radionuclides (SLRs) in the early solar system (ESS) reflect the nucleosynthesis of sources that occurred close to the proto-solar cloud in both space and time. Both the ubiquity of $\mathrm{Sr}$ and $\mathrm{Ba}$ and the diversity of heavy-element abundance patterns observed in single metal-poor stars suggest that some neutron-capture mechanisms other than the $r$-process might have operated in early massive stars. Three such mechanisms are discussed: the weak $s$-process in non-rotating models with initial carbon enhancement, a new $s$-process induced by rapid rotation in models with normal initial composition, and neutron-capture processes induced by proton ingestion in non-rotating models. In addition, meteoritic data are discussed to constrain the core-collapse supernova (CCSN) that might have triggered the formation of the solar system and provided some of the SLRs in the ESS. If there was a CCSN trigger, the data point to a low-mass CCSN as the most likely candidate. An 11.8 $M_{\odot}$ CCSN trigger is discussed. Its nucleosynthesis, the evolution of its remnant, and the interaction of the remnant with the proto-solar cloud appear to satisfy the meteoritic constraints and can account for the abundances of the SLRs ${ }^{41} \mathrm{Ca},{ }^{53} \mathrm{Mn}$, and ${ }^{60} \mathrm{Fe}$ in the ESS.
\end{abstract}

\section{Data on Metal-Poor Stars}

Observations show that heavy elements such as $\mathrm{Sr}$ (with mass numbers $A \sim 86-88$ ) and $\mathrm{Ba}$ ( $A \sim 134-138)$ are prevalent in metal-poor stars [1]. At the early times when these stars were formed, low-mass stars would not have had time to evolve and produce such elements through the slow $(s)$ neutron-capture process. Massive stars evolve quickly to end in core-collapse supernovae (CCSNe) and the neutrino-driven winds from the proto-neutron stars created in these events can produce Sr. Typical winds, however, do not have the appropriate conditions to make Ba (e.g., [2,3]). While the rapid $(r)$ neutron-capture process in rare CCSNe (e.g., [4]) or neutron star mergers (e.g., [5]) can produce $\mathrm{Ba}$, it is difficult for such rare events to account for the wide-ranging enrichment at early times as indicated by the ubiquity of $\mathrm{Ba}$ in metal-poor stars (e.g., [6, 7]). Therefore, some other neutron-capture processes associated with massive stars are required.

Observations also show diverse abundance patterns of heavy elements in metal-poor stars. For example, there are substantial deviations of the abundance ratio $\mathrm{La} / \mathrm{Eu}(A=139$ for $\mathrm{La}$

\footnotetext{
*e-mail: qianx007@umn.edu
} 
and $A=151,153$ for $\mathrm{Eu}$ ) from the solar $r$-process value for stars with metallicities as low as $[\mathrm{Fe} / \mathrm{H}]=\log (\mathrm{Fe} / \mathrm{H})-\log (\mathrm{Fe} / \mathrm{H})_{\odot} \sim-2.6$ [8]. This diversity also calls for massive stellar sources for neutron-capture processes other than the $r$-process.

All of the above observations concern single metal-poor stars. So both the ubiquity of Ba and the diversity of $\mathrm{La} / \mathrm{Eu}$ in these stars reflect enrichment of the general interstellar medium (ISM) by massive stellar sources, not some localized surface contamination through mass transfer of e.g., the $s$-process products from binary companions.

\section{Neutron-Capture Processes in Early Massive Stars}

We focus on three types of neutron-capture processes in early massive stars to address the above observations.

\subsection{Enhanced Weak $s$-Process}

Observations (e.g., [9]) show that there exist carbon-enhanced metal-poor (CEMP) stars with $[\mathrm{C} / \mathrm{Fe}] \geq 0.7$, some of which do not have any enhancements of neutron-capture elements. These so-called CEMP-no stars constitute 20\%, 40\%, and $80 \%$ of all the metal-poor stars with $[\mathrm{Fe} / \mathrm{H}] \leq-2,-3$, and -4 , respectively. Such stars were most likely formed out of the general ISM that had been enriched by very early CCSNe with elevated abundances of C (and presumably also $\mathrm{N}$ and $\mathrm{O}$ ) relative to Fe. While such stars have low masses in order to survive until the present, they must have been formed along with massive CEMP stars that already exploded as CCSNe.

During core $\mathrm{H}$ burning of a massive star, the initial CNO nuclei are converted into ${ }^{14} \mathrm{~N}$, which produces ${ }^{22} \mathrm{Ne}$ through ${ }^{14} \mathrm{~N}(\alpha, \gamma){ }^{18} \mathrm{~F}\left(e^{+} v_{e}\right){ }^{18} \mathrm{O}(\alpha, \gamma){ }^{22} \mathrm{Ne}$ during core He burning. The activation of ${ }^{22} \mathrm{Ne}(\alpha, n)^{25} \mathrm{Mg}$ during core He burning and subsequent evolution provides neutrons for the weak $s$-process that produces heavy elements typically up to Sr. For massive CEMP stars with initial abundances of $[\mathrm{CNO} / \mathrm{H}] \approx-0.5$, even heavier elements up to $\mathrm{Ba}$ can be produced [10]. In general, the efficiency of the $s$-process in massive CEMP stars is sensitive to the initial enhancement of $\mathrm{CNO}$ and mass of the star while the yield increases approximately linearly with the initial Fe abundance. These results were obtained for nonrotating stars, and therefore, differ from similar results for fast-rotating massive metal-poor stars without $\mathrm{C}$ enhancement (e.g., $[11,12])$ in that the former are independent of the uncertain rotation-induced mixing processes. The enhanced weak $s$-process in non-rotating CEMP stars of $\gtrsim 20 M_{\odot}$ with $[\mathrm{CNO} / \mathrm{H}] \gtrsim-1.5$ can be an important source for heavy elements in the early Galaxy [10].

\subsection{New $s$-Process Induced by Rapid Rotation}

Massive metal-poor stars rotating above a critical speed can reach the so-called quasichemically homogeneous (QCH) state following core H burning (e.g., [13, 14]). Rotationinduced mixing results in primary production of ${ }^{13} \mathrm{C}$. The subsequent occurrence of ${ }^{13} \mathrm{C}(\alpha, n){ }^{16} \mathrm{O}$ during core He burning provides neutrons for a prolific $s$-process. Depending on the rotation speed and the mass loss rate, elements up to $\mathrm{Bi}(A=209)$ can be produced for progenitors with initial metallicities of $[\mathrm{Fe} / \mathrm{H}] \lesssim-1.5[15]$. This model of the $s$-process in rotating massive metal-poor stars differs from other studies of the $s$-process in such stars (e.g., [12]) because the QCH state was not reached for the latter.

The above model suggests that rapidly-rotating massive metal-poor stars are likely the first sites of the main $s$-process, which is usually associated with slowly-evolving low-mass 
stars. With the above massive stellar sources, the observed early onset of the $s$-process can be explained. In addition, the $s$-process contributions of these sources can account for at least some of the CEMP- $s$ and CEMP- $r / s$ stars with strong enrichments that are attributed to the $s$-process and a mixture of the $r$-process and the $s$-process, respectively [15].

\subsection{Neutron-Capture Processes Induced by Proton Ingestion}

During the last few years of the life of a metal-free or metal-poor star of $\sim 20-30 M_{\odot}$, the He shell becomes convective following the depletion of $\mathrm{C}$ at the center. Some of the protons present at low levels in the outer He shell can be ingested into the inner He shell by convective boundary mixing. Subsequent transport to the hotter region initiates the reaction sequence ${ }^{12} \mathrm{C}(p, \gamma){ }^{13} \mathrm{~N}\left(e^{+} v_{e}\right){ }^{13} \mathrm{C}(\alpha, n){ }^{16} \mathrm{O}$, which provides neutron densities appropriate for driving a nuclear flow intermediate $(i)$ between the $s$-process and the $r$-process, the so-called $i$-process [16]. As a result, elements up to $\mathrm{Bi}$ are produced [17]. Depending on the time available before core collapse, ${ }^{17} \mathrm{O}(\alpha, n){ }^{20} \mathrm{Ne}$ may occur to provide much lower neutron densities typical of the $s$-process to facilitate further neutron capture on the products of the preceding $i$-process. Consequently, the final yield pattern can vary from $s$-like to $r / s$-like [17].

The above model of neutron-capture processes induced by proton ingestion in early nonrotating massive stars have important implications for the abundances of heavy elements in metal-poor stars. First of all, it provides a rapidly-evolving source that occurred frequently to enrich the early ISM with heavy neutron-capture elements. Therefore, it can account for the observed ubiquity of $\mathrm{Ba}$ in metal-poor stars (see Sect. 1). Further, because its yield pattern can vary from $s$-like to $r / s$-like, it can also explain the diversity of heavy-element abundance patterns in metal-poor stars (see Sect. 1). Finally, mass transfer of $s$-process and $i$-process products from an intermediate-mass binary companion is commonly invoked to account for CEMP- $s$ and CEMP- $r / s$ stars, respectively. For some CEMP- $s$ and CEMP- $r / s$ stars, however, there are no clear indications that they are in binaries. In fact, some CEMP- $s$ stars are observed to be single [18], which is difficult for the binary mass transfer scenario to explain. Single CEMP- $s$ and CEMP- $r / s$ stars, however, can be accounted for by the above model because they could have been formed directly from the early ISM that had already been enriched by massive stars with neutron-capture processes induced by proton ingestion.

\section{CCSN Contributions to the Solar System}

In contrast to metal-poor stars formed at early times, both low-mass and massive stars contributed to the elemental abundances in the solar system, which was formed $\sim 9 \mathrm{Gyr}$ after the big bang. The number of CCSNe among these contributing sources can be estimated as follows. A CCSN can enrich $\sim 3 \times 10^{4} M_{\odot}$ of ISM (e.g., [19]). For a Galactic CCSN rate of $\sim(30 \mathrm{yr})^{-1}$ associated with $\sim 10^{10} M_{\odot}$ of gas, an average ISM would receive CCSN contributions once every $\sim 10 \mathrm{Myr}$. So the ISM out of which the solar system was formed, the proto-solar cloud, received contributions from $\sim 900$ CCSNe over $\sim 9 \mathrm{Gyr}$.

Many short-lived radionuclides (SLRs) with lifetimes of $\sim 0.1-10 \mathrm{Myr}$ are produced by CCSNe. Based on the above estimated interval of $\sim 10$ Myr between successive CCSN contributions, the last few CCSNe contributing to the abundances in the solar system might have provided a significant amount of SLRs to the early solar system (ESS). Any SLRs from those CCSNe would have been incorporated into the meteorites formed in the ESS. Studies of such meteoritic samples indeed found a number of SLRs (e.g., [20]). These meteoritic data provide a strong constraint on the scenario that the last contributing CCSN also triggered the formation of the solar system because the SLRs contributed by that CCSN cannot exceed the corresponding abundances in the ESS. 
Table 1. Example results on SLRs in the ESS.

\begin{tabular}{lccccr}
\hline$R / I$ & $\tau_{R}(\mathrm{Myr})$ & $Y_{R}\left(M_{\odot}\right)$ & $X_{I, \odot}$ & $\left(N_{R} / N_{I}\right)_{\mathrm{ESS}}$ & Data \\
\hline${ }^{41} \mathrm{Ca} /{ }^{40} \mathrm{Ca}$ & 0.147 & $7.11 \times 10^{-6}$ & $5.88 \times 10^{-5}$ & $4.31 \times 10^{-9}$ & $(4.6 \pm 1.9) \times 10^{-9}$ \\
${ }^{53} \mathrm{Mn} /{ }^{55} \mathrm{Mn}$ & 5.40 & $2.73 \times 10^{-5}$ & $1.29 \times 10^{-5}$ & $6.97 \times 10^{-6}$ & $(7 \pm 1) \times 10^{-6}$ \\
${ }^{60} \mathrm{Fe} /{ }^{56} \mathrm{Fe}$ & 3.78 & $4.17 \times 10^{-6}$ & $1.12 \times 10^{-3}$ & $1.04 \times 10^{-8}$ & $(1.01 \pm 0.27) \times 10^{-8}$ \\
\hline
\end{tabular}

For a fraction $f$ of the ejecta from the triggering CCSN injected into the proto-solar cloud and a time $\Delta$ between the CCSN and incorporation of SLRs into the ESS solids, the resulting number ratio of an SLR $(R)$ to its stable isotope $(I)$ would be

$$
\left(\frac{N_{R}}{N_{I}}\right)_{\mathrm{ESS}}=\frac{f Y_{R} / A_{R}}{X_{I, \odot} M_{\odot} / A_{I}} \exp \left(-\frac{\Delta}{\tau_{R}}\right),
$$

where $Y_{R}$ is the CCSN yield of $R, \tau_{R}$ and $A_{R}$ are its lifetime and mass number, respectively, and $X_{I, \odot}$ and $A_{I}$ are the solar abundance and mass number of $I$, respectively. The necessary condition for the triggering CCSN is that $\left(N_{R} / N_{I}\right)_{\mathrm{ESS}}$ match the data on some SLRs without exceeding the data on all the other SLRs.

An additional meteoritic constraint on the triggering CCSN comes from the lack of large deviations in the number ratios of stable isotopes of such major elements as $\mathrm{Mg}, \mathrm{Si}, \mathrm{Ca}$, and Fe [21]. Both this constraint and the necessary condition regarding SLRs render it difficult for a high-mass CCSN to be the trigger because the large yields of such a source would have exceeded the data on some SLRs and caused large deviations in the number ratios of stable isotopes of some major elements [22]. Consequently, if a CCSN triggered the formation of the solar system, it was most likely at the lower end of the relevant mass range [22, 23].

\section{Low-Mass CCSN Trigger for Solar System Formation}

The nucleosynthesis of an $11.8 M_{\odot}$ CCSN was studied in [22, 23]. Using a 3D model of the explosion, [23] found that the SLRs ${ }^{41} \mathrm{Ca},{ }^{53} \mathrm{Mn}$, and ${ }^{60} \mathrm{Fe}$ in the ESS can be accounted for by such a CCSN (see Table 1) with $f=3.6 \times 10^{-6}$ and $\Delta=0.675 \mathrm{Myr}$ [see Eq. (1)]. Because only a very small fraction of the CCSN ejecta needs to have been injected into the proto-solar cloud, the contributions to the other SLRs and the stable isotopes were negligible.

The parameters $f$ and $\Delta$ for the above low-mass CCSN trigger are consistent with the evolution of a CCSN remnant in an ISM and with the interaction between the associated shock and the proto-solar cloud. Simulations showed that a fraction $\epsilon_{\mathrm{in}} \sim 0.05$ of the CCSN ejecta impacting the cloud would be injected into the cloud $[24,25]$. The fraction $f$ of the net CCSN ejecta injected into a cloud of radius $r_{c} \sim 0.1 \mathrm{pc}[26]$ can be estimated as

$$
f \sim \epsilon_{\text {in }}\left(\frac{\delta \Omega_{c}}{4 \pi}\right) \sim \frac{\epsilon_{\text {in }}}{4}\left(\frac{r_{c}}{R_{s}}\right)^{2} \sim 1.25 \times 10^{-4}\left(\frac{\epsilon_{\text {in }}}{0.05}\right)\left(\frac{r_{c}}{0.1 \mathrm{pc}}\right)^{2}\left(\frac{\mathrm{pc}}{R_{s}}\right)^{2},
$$

where $\delta \Omega_{c}$ is the solid angle subtended by the cloud from the center of the CCSN and $R_{S}$ is the shock radius upon impact. To obtain $f \sim 3.6 \times 10^{-6}$ requires $R_{s} \sim 5.9 \mathrm{pc}$. In addition, to trigger the collapse of the cloud requires a shock velocity $v_{s} \sim 20-40 \mathrm{~km} \mathrm{~s}^{-1}[24,25]$.

The evolution of $R_{s}$ and $v_{s}$ for a CCSN remnant [27] can be estimated as

$$
\begin{aligned}
& R_{s}=14.0\left(\frac{E}{10^{51} \mathrm{erg}}\right)^{2 / 7}\left(\frac{\mathrm{cm}^{-3}}{n_{0}}\right)^{3 / 7}\left(\frac{4}{3} t_{*}-\frac{1}{3}\right)^{3 / 10} \mathrm{pc}, \\
& v_{s}=413\left(\frac{E}{10^{51} \mathrm{erg}}\right)^{1 / 14}\left(\frac{n_{0}}{\mathrm{~cm}^{-3}}\right)^{1 / 7}\left(\frac{4}{3} t_{*}-\frac{1}{3}\right)^{-7 / 10} \mathrm{~km} \mathrm{~s}^{-1},
\end{aligned}
$$


where $E$ is the CCSN explosion energy, $n_{0}$ is the hydrogen number density of the ISM, and $t_{*}=t / t_{\text {unit }}$ is the time $t$ since the explosion in units of

$$
t_{\text {unit }}=1.33 \times 10^{4}\left(\frac{E}{10^{51} \mathrm{erg}}\right)^{3 / 14}\left(\frac{\mathrm{cm}^{-3}}{n_{0}}\right)^{4 / 7} \mathrm{yr} .
$$

For $E=2 \times 10^{50} \mathrm{erg}$ [28] and $n_{0} \sim 50 \mathrm{~cm}^{-3}$ [26], $R_{S} \sim 5.9 \mathrm{pc}$ is reached for $t \sim 5.3 \times 10^{4} \mathrm{yr}$ with an impact velocity $v_{s} \sim 33 \mathrm{~km} \mathrm{~s}^{-1}$. Because the above evolution time is far less than $\Delta$, the latter would be essentially the time needed for condensation of solids in the ESS.

\section{Discussion and Conclusions}

Observations of single metal-poor stars reveal both the ubiquity of $\mathrm{Sr}$ and $\mathrm{Ba}$ and the diversity of heavy-element abundance patterns, which appear to require some neutron-capture mechanisms other than the $r$-process to have operated in early massive stars. Among various possibilities, perhaps the weak $s$-process in non-rotating massive CEMP stars is the best understood. Its contributions might have been significant at low metallicities if a substantial fraction of the stars of $\gtrsim 20 M_{\odot}$ had been formed with [CNO/H] $\gtrsim-1.5$ [10]. The new $s$-process induced by rapid rotation can potentially account for the observed early onset of the $s$-process and at least some of the CEMP- $s$ and CEMP- $r / s$ stars [15]. The modeling of this process depends on our understanding of the evolution of rapidly-rotating metal-poor stars and the associated mixing. Likewise, the neutron-capture processes induced by proton ingestion in early non-rotating massive stars depend on the treatment of mixing during the evolution of such stars. These processes can potentially account for both the observed ubiquity of $\mathrm{Ba}$ and the diversity of heavy-element abundance patterns in metal-poor stars [17]. In addition, their relatively frequent contributions to the ISM may have resulted in the formation of a significant fraction of the CEMP- $s$ and CEMP- $r / s$ stars by birth, which could have been the dominant channel to form such stars in single configuration [17].

Meteoric data demonstrate the presence of SLRs in the ESS. They also show a lack of large deviations in the number ratios of stable isotopes of such major elements as $\mathrm{Mg}, \mathrm{Si}, \mathrm{Ca}$, and Fe. These data render it difficult for a high-mass CCSN to be the trigger for the formation of the solar system because the large yields of such a source would have exceeded the data on some SLRs and caused large deviations in the number ratios of stable isotopes of some major elements [22]. Consequently, if there was a CCSN trigger, a low-mass CCSN would be the most likely candidate $[22,23]$. The nucleosynthesis of an $11.8 M_{\odot}$ CCSN, the evolution of its remnant, and the interaction of the remnant with the proto-solar cloud appear to satisfy the meteoritic constraints and can account for the abundances of the SLRs ${ }^{41} \mathrm{Ca},{ }^{53} \mathrm{Mn}$, and ${ }^{60} \mathrm{Fe}$ in the ESS.

\section{Acknowledgments}

I thank Projjwal Banerjee, Wick Haxton, Alexander Heger, Bernhard Müller, and Andre Sieverding for collaboration. This work was supported in part by the U.S. Department of Energy under Grant No. DE-FG02-87ER40328.

\section{References}

[1] I. U. Roederer, Astron. J. 145, 26 (2013).

[2] Y.-Z. Qian and S. E. Woosley, Astrophys. J. 471, 331 (1996). 
[3] R. D. Hoffman, S. E. Woosley, and Y.-Z. Qian, Astrophys. J. 482, 951 (1997).

[4] T. Fischer, M.-R. Wu, B. Wehmeyer, N.-U. F. Bastian, G. Martínez-Pinedo, and F.-K. Thielemann, Astrophys. J. 894, 9 (2020).

[5] F.-K. Thielemann, M. Eichler, I. V. Panov, and B. Wehmeyer, Annu. Rev. Nucl. Part. Sci. 67, 253 (2017).

[6] Y.-Z. Qian, Astrophys. J. 534, L67 (2000).

[7] D. Argast, M. Samland, F.-K. Thielemann, and Y.-Z. Qian, Astron. Astrophys. 416, 997 (2004).

[8] J. Simmerer, C. Sneden, J. J. Cowan, J. Collier, V. M. Woolf, and J. E. Lawler, Astrophys. J. 617, 1091 (2004).

[9] W. Aoki, T. C. Beers, N. Christlieb, J. E. Norris, S. G. Ryan, and S. Tsangarides, Astrophys. J. 655, 492 (2007).

[10] P. Banerjee, Y.-Z. Qian, and A. Heger, Mon. Not. R. Astron. Soc. 480, 4963 (2018).

[11] M. Pignatari, R. Gallino, G. Meynet, R. Hirschi, F. Herwig, and M. Wiescher, Astrophys. J. 687, L95 (2008).

[12] U. Frischknecht et al., Mon. Not. R. Astron. Soc. 456, 1803 (2016).

[13] S.-C. Yoon and N. Langer, Astron. Astrophys. 443, 643 (2005).

[14] S. E. Woosley and A. Heger, Astrophys. J. 637, 914 (2006).

[15] P. Banerjee, A. Heger, and Y.-Z. Qian, Astrophys. J. 887, 187 (2019).

[16] J. J. Cowan and W. K. Rose, Astrophys. J. 212, 149 (1977).

[17] P. Banerjee, Y.-Z. Qian, and A. Heger, Astrophys. J. 865, 120 (2018).

[18] T. T. Hansen et al., Astron. Astrophys. 588, A3 (2016).

[19] K. Thornton, M. Gaudlitz, H. -Th. Janka, and M. Steinmetz, Astrophys. J. 500, 95 (1998).

[20] A. M. Davis and K. D. Mckeegan, Meteorites and Cosmochemical Processes, Treatise on Geochemistry vol. 1 (Elsevier, 2014) 361-395.

[21] G. J. Wasserburg, M. Busso, R. Gallino, and K. M. Nollett, Nucl. Phys. A777, 5 (2006).

[22] P. Banerjee, Y.-Z. Qian, A. Heger, and W. C. Haxton, Nat. Commun. 7, 13639 (2016).

[23] A. Sieverding, B. Müller, and Y.-Z. Qian, Astrophys. J. 904, 163 (2020).

[24] A. P. Boss and S. A. Keiser, Astrophys. J. 788, 20 (2014).

[25] A. P. Boss and S. A. Keiser, Astrophys. J. 809, 103 (2015).

[26] E. A. Bergin and M. Tafalla, Annu. Rev. Astron. Astrophys. 45, 339 (2007).

[27] D. F. Cioffi, C. F. McKee, and E. Bertschinger, Astrophys. J. 334, 252 (1988).

[28] B. Müller et al., Mon. Not. R. Astron. Soc. 484, 3307 (2019). 